\title{
BIODIVERSIDADE DE MOSCAS CALLIPHORIDAE NO LIXÃO URBANO DE PRESIDENTE PRUDENTE, SÃO PAULO, BRASIL
}

\author{
L. Seolin Dias ${ }^{1 *}$, V.A. Santarém ${ }^{1 *}$, M.S.R. Almeida ${ }^{1}$, A.O. Medina ${ }^{1}$, A.V. da Silva ${ }^{2}$ \\ ${ }^{1}$ Universidade do OestePaulista, Rod. Raposo Tavares, km 572,CEP19067-175, PresidentePrudente, SP, Brasil. \\ E-mail:nseolin@terra.com.br
}

\section{RESUMO}

Com o objetivo de estudar a biodiversidade de califorídeos no lixão de Presidente Prudente, São Paulo, Brasil, foram alocadas seis armadilhas confeccionadas com garrafas tipo "pet", no período de março de 2006 a março de 2007, ao redor do lixão. Utilizou-se como atrativo, aproximadamente, $250 \mathrm{~g}$ de fígado bovino, com a troca semanal das armadilhas. Foram capturadas 44.688 califorídeos, com maior frequência das espécies Chrysomya megacephala $(93,61 \%$; $\mathrm{n}=41833)$, seguindo-se de $C$. albiceps $(1,5 \% ; n=672)$ e C. putoria $(0,79 \% ; n=352)$, com diferença significativa entre a primeira e as demais espécies $(p<0,05)$. Houve influência da temperatura e da precipitação pluviométrica na sazonalidade de $C$. megacephala, com maiores capturas nos meses quentes e chuvosos $(\mathrm{p}<0,05)$. Nos meses frios, a captura das moscas, especialmente de C. albiceps e de C. putoria, foi praticamente ausente. Dessa forma, conclui-se que o depósito de lixo urbano de Presidente Prudente oferece condições para a manutenção de moscas varejeiras, especialmente C. megacephala.

PALAVRAS-CHAVE: Chrysomya megacephala, lixão, epidemiologia.

\section{ABSTRACT}

BIODIVERSITY OF CALLIPHORIDAE FLIES FROM URBAN GARBAGE DUMPS OF PRESIDENTE PRUDENTE, SÃO PAULO, BRAZIL. In order to study the biodiversity of Calliphoridae in the of garbage site of Presidente Prudente, São Paulo, Brazil, six traps made apart plastic pet bottles, were disposed surrounding the garbage. The study was carried out between March, 2006 and March, 2007. Bovine liver was used as bait, and the bottle were changed weekly. The total of Calliphoridae captured was 44,688 . Chrysomya megacephala was the most frequent species $(93.61 \%$; $\mathrm{n}=41,833)$ of fly, with significant differences $(\mathrm{p}<0.05)$ between $C$. megacephala and C. albiceps $(1.50 \%$; $\mathrm{n}=672)$ and C. putoria $(0.79 \% ; \mathrm{n}=352)$. There was influence of temperature and of rainfall on the C. megacephala population. In the coldest months, the capture of flies, especially C. albiceps e de $C$. putoria was extremely low. Regarding the Muscidae family, 1307 individuals were captured. Thus, it is concluded that the garbage site of Presidente Prudente is an environment that gives conditions to maintenance of Calliphoridae, especially C. megacephala.

KEY WORDS: Chrysomya megacephala, dump, epidemiology.

\section{INTRODUÇÃO}

Os resíduos de sólidos urbanos, mais conhecidos como lixo, constituem uma preocupação ambiental mundial, especialmente em grandes centros urbanos de países subdesenvolvidos (REGO et al., 2002).

Fatores como crescimento demográfico, melhora do nível sócio-econômico da população, desenvolvimento denovos hábitos e intensificação do consumo, além de provocarem modificações nas características dos resíduos sólidos gerados, acabam por trazer difi- culdades técnicas e operacionais para a sua correta destinação final e o respectivo tratamento (PRADO FILHO; SOBREIRA, 2007).

A maior parte dos resíduos recolhidos nas cidades é destinada de forma inadequada em depósitos a céu aberto existentes nas periferias das grandes cidades, denominados de lixões. Esses biótopos, ricos em matéria orgânica, dealto teor energético, possibilitam a proliferação de insetos, especialmente de moscas, como da família Calliphoridae(Lima, 1986; KopP, 1988; Branco, 1997; D'Almeida; VilHena, 2000).

${ }^{2}$ Universidade Paranaense, Umuarama, PR, Brasil.

*Mestrado em Ciência Animal - Universidade do Oeste Paulista (Unoeste). 
Algumas espécies dessa família despertam grande interesse médico e sanitário, uma vez que são potenciais vetores mecânicos de agentes patógenos como: vírus, bactérias, cistos de protozoários e ovose larvas de helmintos, cistos de protozoários e enterovírus (GREENBERG, 1971; LiMA; LuZ, 1991; BARRIGA, 2002), e podem causar míases no homem e nos animais (ZUMPT, 1965; GUIMARÃEs et al., 1983).

Essas moscas, por suas características sinantrópicas, podem se proliferar em diversos ambientes, inclusivenos depósitos de lixo urbano. Entretanto, há uma escassez de estudos sobre a biodiversidade desses insetos nesse meio (LIMA; LuZ, 1991; LOPES, 2000; Mello et al., 2004; SEOLIN DiAs; SARTOR, 2005).

Considerando esses fatos, realizou-se o presente trabalho cujo objetivo foi avaliar a biodiversidade populacional de moscas da família Calliphoridae no lixão de Presidente Prudente, São Paulo.

\section{MATERIALEMÉTODOS}

As capturas dos dípteros foram realizadas semanalmente, no período de março de 2006 a março de 2007, no lixão de Presidente Prudente, situado a cerca de seis quilômetros do centro da cidade, com área aproximada de $90.000 \mathrm{~m}^{2}$ e perímetro de $1,36 \mathrm{~km}$.

Para a coleta foram alocadas seis armadilhas confeccionadas com garrafas tipo "pet" de dois litros (CUNHA; LOMÔNACO, 1996) ao redor do lixão, dispostas em árvores a uma altura de 1,0 a 1,70 m do solo.

Comoisca, utilizou-se aproximadamente $250 \mathrm{~g}$ de fígado bovino para cada armadilha (VIANNA et al., 2004). A cada captura, as garrafas e o atrativo foram substituídos, e as moscas coletadas transferidas para um frasco de vidro contendo álcool 70\%. Quando necessário, um inseticida aerossolà base de piretroide foi aplicado no interior da armadilha para matar os insetos (GOMEs et al., 2000).

As moscas foram dispostas em placas de Petri e submetidas à secagem em estufa $50^{\circ} \mathrm{C}$, de 30 a 40 minutos, a depender do número de espécimes capturados, com a finalidade de facilitar a sua visualização, que foi realizada com auxílio de microscópio estereoscópio, e identificação com base na chave dicotômica das espécies (CARVALHO; RIBEIRO, 2000).

Embora sejam necessários pelo menos três anos para determinar a flutuação sazonal de populações de moscas, nesse estudo decidiu-se avaliar a influência da temperatura e precipitação pluviométrica sobre a dinâmica populacional.

Os registros climáticos semanais foram fornecidos pelo Centro de Meteorologia da Universidade do Oeste Paulista, Unoeste, Presidente Prudente, São Paulo, latitude $22^{\circ} 07^{\prime} \mathrm{S}$ e longitude $51^{\circ} 22^{\prime} \mathrm{W}$. O muni- cípio está a 424,29 m acima do nível do mar e tem temperatura média de $23,1^{\circ} \mathrm{C}$.

Para comparação da frequência de espécies Calliphoridae, os dados foram submetidos ao teste $t$ deStudent. Através da análise de correlação linear de Pearson, estudou-se a influência da temperatura e precipitação pluviométrica sobre a flutuação populacional das espécies. Adotou-se como nível de significância estatística $\mathrm{p}<0,05$.

\section{RESULTADOSEDISCUSSÃO}

Das moscas Calliphoridae capturadas no lixão de Presidente Prudente, as espécies mais frequentes foram Chrysomya megacephala, C. albiceps e C. putoria (Tabela 1), havendo diferença significativa da população de $C$. megacephala em relação às outras duas espécies.

Essas moscas são originárias do Velho Mundo e da África, e foram introduzidas na década de 1970 no Estado do Paraná. Essas espécies apresentam um alto potencial de adaptação e colonização em novos ambientes, o que resultou na redução significativa das populações de moscas nativas e na sua rápida dispersão por todo o território nacional (GUIMARÃes et al., 1979).

Essas espécies, que são amplamente registradas em ambientes rurais e urbanos (MADEIRA et al., 1989), têm sido descritas como as mais frequentemente encontradas em depósitos delixo urbanos. No depósito de lixo de Curitiba (Lima; Luz, 1991), C. putoria foi a mais abundante, com 70,59\%. No Rio de Janeiro, com $1,96 \%$ dos insetos capturados, essa espécie representou a sexta posição; e, C. albiceps a segunda, com 23,11\% do total coletados (Mello et al., 2004). C. putoria foi a mais populosa no aterro sanitário de Curitiba, porém com uma frequência de 57,32\%(LimA; LuZ, 1991). Em Goiânia (FERREIRA; LACERDA, 1993) e Rio de Janeiro (Melloet al., 2004), C. megacephala foi a mais frequente $(48,19 \%$ e $55,98 \%$, respectivamente) nesse biótopo.

Tabela 1 - Prevalência de moscas Calliphoridae no lixão de Presidente Prudente, São Paulo, 2006-2007.

\begin{tabular}{lcc}
\hline & \multicolumn{2}{c}{ Abundância } \\
\cline { 2 - 3 } Espécies & Absoluta & Relativa (\%) \\
\hline Chrysomya megacephala & $41.833^{\mathrm{A}}$ & 93,61 \\
Chrysomya albiceps & $672^{\mathrm{B}}$ & 1,50 \\
Chrysomya putoria & $352^{\mathrm{B}}$ & 0,79 \\
Outras Calliphoridae & 1.831 & 4,1 \\
\hline
\end{tabular}

*Letras diferentes indicam em colunas diferença significativa entre as médias de moscas capturadas $(\mathrm{p}<0,05)$. 
A flutuação de moscas Calliphoridae no lixão de Presidente Prudente está disposta na Figura 1. C. megacephala foi observada durante todo o período, com maior frequência nos meses de verão, quando se capturou 29.951 espécimes. A partir de abril, houve decréscimo dessas moscas, com menor contagem em julho. Esse resultado é semelhante ao observado no aterro de Goiânia (LIMA; Luz, 1991).

Em relaçãoà C.albiceps houve picono mês deabril. Nãohouve, entretanto, captura entrejulho a setembro e em dezembro. A maior abundancia de C. putoria ocorreu no verão, não havendo registro nos meses de julho, setembro e dezembro.

Na análise de correlação(Tabelas 2e3), observouse que C. megacephala foi a espécie que sofreu influên- cia da temperatura e da pluviosidade. Ou seja, o número de moscas foi diretamente proporcional a esses fatores climáticos.

Nesse estudo, diferentemente do que ocorreu em outras pesquisas, onde a população de $C$. albiceps e $C$. putoria esteve presenteem todoo período deverão,não houve captura de nenhum espécime dessas moscas em dezembro. Nestemês ocorreu um volumedechuva acima do esperado para região, 258,6 mm, uma vez que a média histórica da Cidade de Presidente Prudenteéde $210 \mathrm{~mm}$, segundoo Centro de Meteorologia da Unoeste.

Segundo Dajoz (1983) e Vianna et al. (2004), as intempéries climáticas são mais importantes no equilíbrio da população de califorídeos, enquanto os fatores bióticos exercem papel secundário.
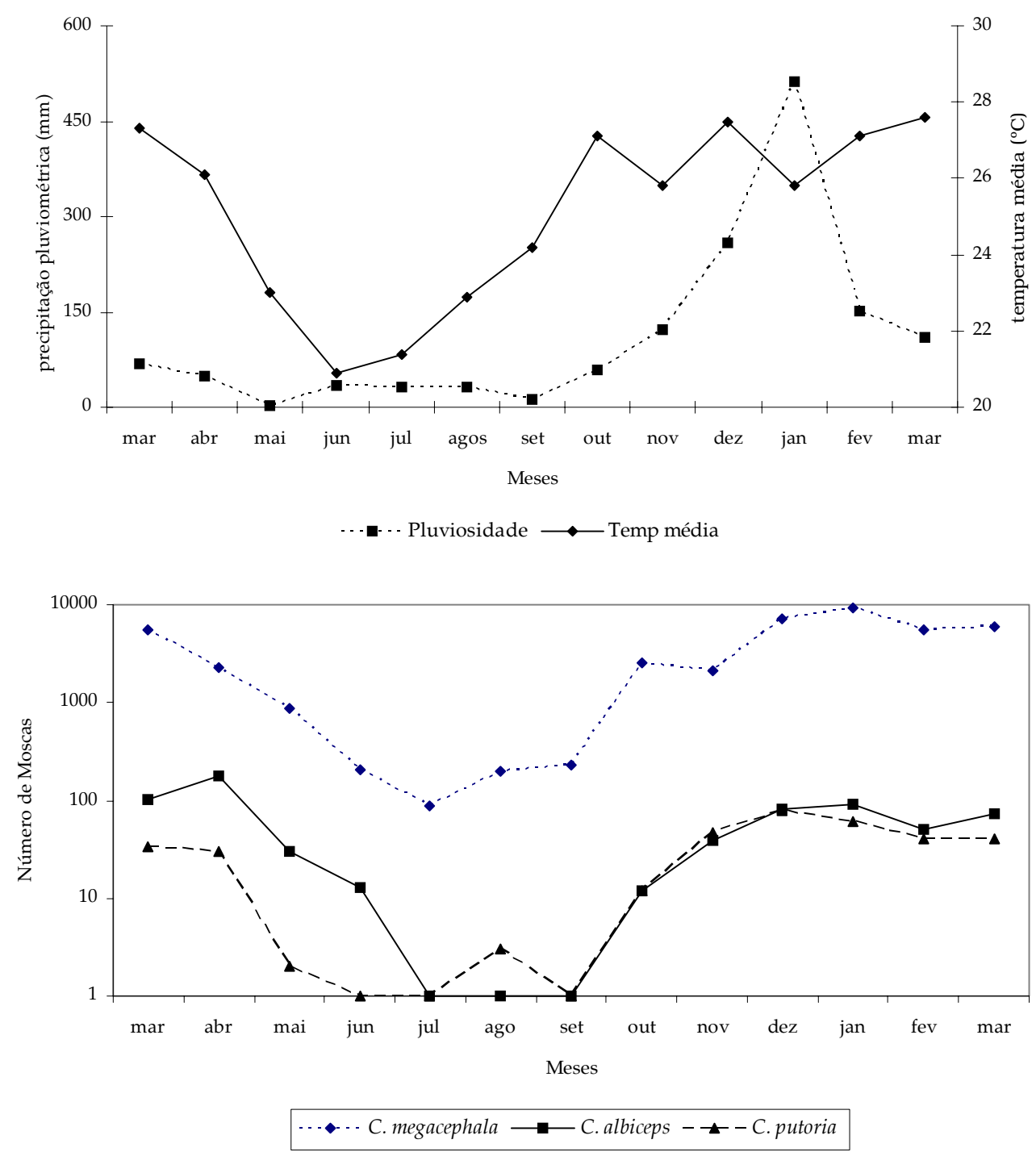

Fig. 1 - Dinâmica populacional das espécies de Calliphoridae (Chrysomya megacephala, C. albiceps e C. putoria) no período de março de 2006 a março de 2007, no lixão de Presidente Prudente, São Paulo, Brasil, e variação das médias mensais de precipitação pluviométrica e temperatura do município. 
Tabela 2 - Coeficientes de correlação (r), estimativa do intervalo de confiança a 95\% para r (IC95\%), coeficiente de determinação $\left(\mathrm{r}^{2}\right)$ e valor de $\mathrm{P}$, entre o número de moscas, segundo a espécie, e a temperatura média em um período de 13 meses. Presidente Prudente, SP. 2006-2007.

\begin{tabular}{lcccc}
\hline Espécie de Calliphoridae & Coeficiente de correlação $(\mathrm{r})$ & $\mathrm{IC} 95 \%$ & $\mathrm{r}^{2}$ & $\mathrm{P}$ \\
\hline Chrysomya megacephala & 0,6048 & $0,0805-0,8669$ & 0,3658 & 0,0285 \\
C. albiceps & 0,0004 & $-0,5508-0,5513$ & $1,25.10^{-7}$ & 0,9991 \\
C. putoria & 0,4975 & $-0,0738-0,8230$ & 0,2475 & 0,0836 \\
Outras & 0,0534 & $-0,5127-0,5872$ & 0,0028 & 0,8623 \\
\hline
\end{tabular}

Tabela 3 - Coeficientes de correlação (r), estimativa do intervalo de confiança a 95\% para r (IC95\%), coeficiente de determinação $\left(\mathrm{r}^{2}\right)$ e valor de $\mathrm{P}$, entre o número de moscas Calliphoridae, segundo a espécie, e a taxa de precipitação pluviométrica em um período de 13 meses. Presidente Prudente, SP. 2006-2007.

\begin{tabular}{lccrc}
\hline Espécie de Calliphoridae & Coeficiente de correlação (r) & IC 95\% & $\mathrm{r}^{2}$ & $\mathrm{P}$ \\
\hline Chrysomya megacephala & 0,8281 & $0,5096-0,9470$ & 0,6858 & 0,0005 \\
C. albiceps & 0,0893 & $-0,4857-0,6103$ & 0,0080 & 0,7718 \\
C. putoria & 0,5001 & $-0,0704-0,8241$ & 0,2501 & 0,0818 \\
Outras & $-0,2245$ & $-0,6902-0,3727$ & 0,0504 & 0,4608 \\
\hline
\end{tabular}

Trabalhos brasileiros realizados em outros ambientes que não os depósitos de resíduos urbanos, como granja avícola de Uberlândia, MG (CuNHA; LOMÔNACO, 1996), campus universitário (RODRIGUES-GUIMARÃES et al., 2001), fragmento de mata e (LEANDRO; D'ALMEIDA, 2005) e reserva biológica do Rio de Janeiro(MARINHOet al.,2006) e em área urbana de Pelotas, RS(VIANNA et al., 2004), edeRio deJaneiro, RJ (MarinHoet al., 2003), eem zona rural e florestal de Campinas (LINHARES, 1979), e de Rio de Janeiro (Mello et al., 2004; RODRIGUES-GUIMARÃEs et al., 2007) e de Presidente Prudente, SP (Moço et al., 2007), apontam que a flutuação dos califorídeos está estritamente associada às condições ambientais.

Algumas medidas podem ser adotadas para redução de moscas sinantrópicas em lixões, como a implantação de programas de coleta seletiva e criação de aterro sanitário, que visam a diminuição de matéria orgânica que serve como substrato para a sobrevivência desses insetos (SEOLIn Dias et al., 2005).

\section{CONCLUSÕES}

As informações apresentadas no estudo mostram que as condições ambientais do depósito de lixo urbano de Presidente Prudente são favoráveis à proliferação de califorídeos, especialmente de C. megacephala.

Cabe à autoridade sanitária, por intermédio dos órgãos competentes, uma destinação adequada do lixo domiciliar e de outros resíduos de qualquer natureza, visto que a presença de um lixão representa risco para a saúde pública, contribuindo para a transmissão de doenças e de seus vetores.

\section{AGRADECIMENTOS}

À Universidade do Oeste Paulista (Unoeste)- PPD 139/06. À Prudenco(CompanhiaPrudentina deDesenvolvimento), àSecretaria doMeioAmbientedePresidente Prudente, ao Centro Meteorológico da Unoeste e ao Frigorífico Estrela (Tupã), pelo apoio e cooperação.

\section{REFERENNCIAS}

BARRIGA, O.O. (Ed.) Las enfermedades parasitarias de los animales domésticos en la America Latina. Santiago do Chile: Editorial Germinal, 2002. 247p.

BRANCO, S.M. Hidrobiologia aplicada à engenharia sanitária. São Paulo: CETESB, 1997.

CARVALHO, C.J.B.; RIBEIRO, P.B. Chave de identificação das espécies de calliphoridae (Diptera) do sul do Brasil. Revista Brasileira de Parasitologia Veterinária, v.9, p.169-173, 2000.

CUNHA, C.L.; LOMÔNACO, C. Monitorização de impacto ambiental provocado por dispersão de moscas em bairros adjacentes a uma granja avícola. Anais da Sociedade Entomológica do Brasil, v.25, p.1-12, 1996.

D'ALMEIDA, M.L.O.; VILHENA, A. (Ed.) Lixo municipal: manual de gerenciamento integrado. 2.ed. São Paulo: IPT/CEMPRE, 2000.

DAJOZ, R. (Ed.) Ecologia geral. 4.ed. Petrópolis: Vozes, 1983. 472p.

FERREIRA, M.J.M.; LACERDA, P.V. Muscóides sinantrópicos associados ao lixo urbano em Goiânia, 
Goiás. Revista Brasileira de Zoologia, v.10, p.185-195, 1993.

GOMES, A.; KOLLER, W.W.; BARROS, A.T.M. Sazonalidade da mosca-varejeira, Cochliomyia macellaria (Diptera: Calliphoridae), na região dos cerrados, Campo Grande, MS. Revista Brasileira de Parasitologia Veterinária, v.9, p.125-128, 2000.

GREENBERG, B. (Ed.). Flies and diseases. Ecology, classification and biotic associations. Princeton: Princeton University, 1971. 856p.

GUIMARÃES, J.H.; PRADO, A.P.; BURALLI, G.M. Dispersal and distribution of three newly introduced species of Chrysomya Robineau-Desvoidy in Brazil (Diptera; Calliphoridae). Revista Brasileira de Entomologia, v.23, p.245-255, 1979.

GUIMARÃES, J.H.; PAPAVERO, N.A.; PRADO, A.P. As miíases na região Neotropical (identificação, biologia e bibliografia). Revista Brasileira de Zoologia, v.1; p.239416, 1983.

KOPP, R.L. Chrysomya megacephala (Fabricius, 1974) (Diptera Calliphoridae) veiculadora de fungos em Curitiba, Paraná. In: CONGRESSO BRASILEIRA DE ZOOLOGIA, 15., 1988, Curitiba, PR. Resumos. Curitiba: 1988. p.229.

LEANDRO, M.J.F.; D'ALMEIDA, J.M. Levantamento de Calliphoridae, Fanniidae, Muscidae e Sarcophagidae em um fragmento de mata na Ilha do Governador, Rio de Janeiro, Brasil. Iheringia, v. 95, p.377-381, 2005. (Série Zoologia).

LIMA, L.M.Q. (Ed.) Tratamento de lixo. São Paulo: Hemus Editora, 1986. 240p.

LIMA, M.L.P.S.; LUZ, E. Espécies exóticas de Chrysomiya, como veiculadoras de enterobactérias patogênicas em Curitiba, Paraná, Brasil. Acta Biológica Paranaense, v.20, p.61-83, 1991.

LINHARES, A.X. Sinantropia de dipteros muscóides de Campinas. 1979. 129f. Dissertação (Mestrado) - Universidade Estadual de Campinas, Campinas, 1979.

LOPES, S.M.A influência de vários fatores abióticos na atratividade de dípteros muscóides em lixo urbano exposto. Revista de Entomologia y Vectores, v.7, p.163$189,2000$.

MADEIRA, N.G.; SILVEIRA, G.A.R.; PAVAN, C. The occurrence of primary myiasis in cats caused by Phaenicia eximia (Diptera: Calliphoridae). Memórias do Instituto Oswaldo Cruz, v.84, p.341, 1989.

MARINHO, C.R.; AZEVEDO, A.C.G.; AGUIARCOELHO, V.M. Diversidade de califorídeos (Diptera: Calliphoridae) em área urbana, Rio de Janeiro. Entomologia y Vectores, v.10, p.185-199, 2003.
MARINHO, C.R.; BARBOSA, L.S.; AZEVEDO, A.C.G.; QUEIROZ, M.M.C.; VALGODE, M.A.; COELHO, V.M.A. Diversity of Calliphoridae (Diptera) in Reserva Biológica do Tinguá, Rio de Janeiro, Brasil. Brazilian Journal of Biology, v.66, p.95-100, 2006.

MELLO, R.P.; GREDILHA, R.; GUIMARÃES-NETO, E.G. Dados preliminares sobre sinantropia de califorídeos (Díptera: Calliphoridae) no municipio de ParacambíRJ. Revista Universidade Rural. Série Ciências da Vida, v.24, p.97-101, 2004.

MOÇO, B.M.; SEOLIN DIAS, L.; OLIVEIRA, N.N.; GUIMARÃES, R.B. Freqüência de moscas no bairro Morada do Sol, do Município de Presidente Prudente, SP. In: ENCONTRO DE ENSINO, PESQUISA E EXTENSÃO, 11., 2007, Presidente Prudente, SP. Resumos. Presidente Prudente, 2007. p.333-335. 1 CD-ROM.

PRADO FILHO, J.F.; SOBREIRA, F.G. Desempenho operacional e ambiental de unidades de reciclagem e disposição final de resíduos sólidos domésticos financiadas pelo ICMS Ecológico de Minas Gerais. Engenharia Sanitária e Ambiente, v.12, p.52-61, 2007.

REGO, R.C.F.; BARRETO, M.L.; KILLINGER, C.L. O que é lixo afinal? Como pensam mulheres residentes na periferia de um grande centro urbano. Cadernos de Saúde Pública, v.18, p.1583-1591; 2002.

RODRIGUES-GUIMARÃES, R.; GUIMARÃES, R.R.; PILE, E.A.M.; NORBERG, A.N.; QUEIROZ, M.M.C. Ocorrência de dípteros califorídeos (Díptera: Calliphoridae) no Campus I da Universidade IguaçuUNIG, Nova Iguaçu, Rio de Janeiro, Brasil. Entomologia y Vectores, v.8, p.245-260, 2001.

RODRIGUES-GUIMARÃES, R.; GUIMARÃES, R.R.; BARROS, H.M.; CARVALHO, R.W.; MOYA-BORJA, G.E. Abundância absoluta, relativa e sazonalidade de dípteros califorídeos (Diptera, Calliphoridae) na baixada fluminense, Rio de Janeiro, Brasil. Revista de Ciência \& Tecnologia, v.6, p.50-63, 2007.

SEOLIN DIAS, L.; SARTOR, F.I. Efeito de um programa de coleta seletiva de lixo na flutuação populacional de dípteros (Calliphoridae e Muscidae). In: FÓRUM AMBIENTAL DA ALTA PAULISTA, 1., 2005, Tupã, SP. Anais. Resumos. Tupã, 2005. 1 CD_ROM.

VIANNA, E.E.S.; COSTA, P.R.P.; FERNANDES, A.L; RIBEIRO, P.B. Abundância e flutuação populacional das espécies de Chrysomya (Díptera, Calliphoridae) em Pelotas, Rio Grande do Sul, Brasil. Iheringia, Série Zoologia, v.94, p.231-234, 2004.

ZUMPT, F. (Ed.). Myiasis in man and animals in the Old World. London: Butterworths, 1965. 267p.

Recebido em 30/7/08

Aceito em 29/6/09 